\title{
Bushenshugan Formula Attenuates the Development of Lung Cancer by Inhibiting Epithelial-Mesenchymal Transition
}

\author{
Zhirui Fan ${ }^{a, b}$ Wenhua Xue ${ }^{d} \quad$ Mengmeng Dou ${ }^{b}$ Lifeng Li ${ }^{a, d} \quad J i n g l i ~ L u^{d}$ \\ Bingjun Ma ${ }^{a} \quad$ Xiaoming Deng ${ }^{b} \quad$ Mingzhi Zhang ${ }^{a}$ Yunkai Zhaie Shuling Wang ${ }^{c}$ \\ Jie Zhaod,e \\ aDepartment of Oncology, the First Affiliated Hospital of Zhengzhou University, Zhengzhou, \\ ${ }^{b}$ Department of Chinese and Western Integrative Medicine, the First Affiliated Hospital of Zhengzhou \\ University, Zhengzhou, 'Basic Medical College of Zhengzhou University, Zhengzhou, 'Department of \\ Pharmacy, the First Affiliated Hospital of Zhengzhou University, Zhengzhou, Internet medical and \\ system applications of National engineering laboratory, Zhengzhou, China
}

\section{Key Words}

Bushenshugan Formula • Epithelial-mesenchymal transition • Lung cancer

\begin{abstract}
Background/Aims: BushenShugan Formula (BSF) is a traditional Chinese medicine that has therapeutic effects on middle- and late-stage lung adenocarcinoma in clinical application. It was reported that Bushen Chinese medicine suppressed the onset of pre-metastatic niches in a murine model of spontaneous lung metastasis. However, the mechanisms of BSF on human lung adenocarcinoma remain unknown. Methods: Cell proliferation was determined by CCK8 and colony formation. Cell apoptosis and cell cycle were detected by flow cytometry. Cancer stem cells properties were examined by spheroid body formation. The migration and invasion abilities were analyzed by wound healing assay and transwell invasion assay. The mRNA expressions were determined by qRT-PCR. Western blotting analysis showed the protein levels. Results: BSF was shown to inhibit the proliferation of A549 cells in time- and concentrationdependent manners. Colony formation assays also indicated the antiproliferative effect of BSF against A549 cells. Cellular mechanistic studies demonstrated that BSF arrested the cell cycle in G2/M phase and induced apoptosis. Importantly, BSF could inhibit the epithelialmesenchymal transition(EMT) of A549 cells through PI3K/AKT/NF-KB pathway. Conclusions: BSF effectively inhibited tumour growth, suggesting that it is a promising anticancer treatment for further clinical development.

Z. Fan, W. Xue and M. Dou contributed equally to this work.

Jie Zhao

and Shuling Wang
Department of Pharmacy, the first Affiliated Hospital of Zhengzhou University

No.1 Jianshe road, Zhengzhou, Henan (China)

Tel. +86 371 66295320, 0371- 66915501, E-Mail jiezhaoz2016@163.com,wsI5869@163.com 


\section{Introduction}

Lung cancer is a malignant tumour that is seriously harmful to human health. The incidence and mortality of lung cancer are the highest among malignant tumours worldwide. Lung cancer is a highly metastasizing cancer and the number one cause of death in humans; it is classified into two broad histologic classes in terms of growth and spread: small-cell lung carcinoma(SCLC)and non-small cell lung carcinomas(NSCLC). Although some earlystage lung cancer patients can be successfully treated, $70 \%$ of patients present with advanced stage when diagnosed, the cancer has already spread locally or to distant organs, and have lost the chance of operation [1]. Metastasis is the leading cause of death by cancer. Chemotherapy has advanced considerably over the last 30 years, but the 5-year relative survival rate for lung cancer remains only $17.4 \%$ [2]. Elucidation of the mechanisms of lung cancer and development of various treatments (surgery, chemotherapy, and radiotherapy) has made advances in recent years, especially for NSCLC. Cisplatin(DDP) is a well-known chemotherapeutic drug for the treatment of NSCLC, but poor compliance and drug resistance remain concerns. Thus, there is an urgent need to develop novel anticancer agents, which may include highly effective Chinese medicines.

EMT is an important regulator in lung cancer cells. In addition, it is also a key process that occasionally occurs during lung cancer metastasis and invasion in which lung cancer cells acquire a more aggressive phenotype $[3,4]$. Based on these significant findings, EMT is present in a variable proportion of lung cancer cells and results in up-regulation of mesenchymal markers, such as $\mathrm{N}$-cadherin, snail and vimentin, and down-regulation of epithelial markers, such as E-cadherin.EMT is an important mechanism of tumour invasion and metastasis, suggesting that EMT may be a key factor in metastasis of lung cancer and providing a new direction for the treatment of lung cancer.

Modern clinical pharmacology research and preliminary results have shown that Chinese medicine has a multi-target effect and can inhibit the recurrence and metastasis of tumour and significantly improve patient quality of life [5-8]. There are many varieties and sources of Chinese medicine in China, and further analysis of the traditional theory of TCM (Traditional Chinese Medicine)and identification of effective anti-tumour monotherapies or compounds based on modern scientific results is one direction for anti-tumour research in the future. Bushen Chinese medicine has been shown to suppress the onset of the premetastatic niche in a murine model of spontaneous lung metastasis [9]. BSF is derived from Wuzi Yanzong pills and composed of Schisandra, Bupleurum, Chinese angelica, Medlar, Dodder seed, Ligustrumlucidum, Plantago, Fructus trichosanthis and 10 other Chinese herbal medicines. BSF has good anti-lung cancer metastasis effects, and it could prevent tumour recurrence, as the clinical empirical formula exhibits potent therapeutic effects on human lung carcinoma in our previous studies [10-13]. However, the mechanisms of BSF on human lung adenocarcinoma and the effect on EMT remain unknown. In this study, we aimed to investigate whether BSF inhibited EMT and explored the possible anticancer mechanisms on human lung cancer.

\section{Materials and Methods}

\section{Animals}

Sixty male SD rats, weighing 250-300g, were supplied by the Center of Experiment Animal of Zhengzhou University [SCXK (Yu) 2010-0002, Henan, China]. The animal experimental protocol was reviewed and approved by the Institutional Animal Care and Use Committee of Zhengzhou University. All rats were maintained under a regulated temperature $\left(18-22^{\circ} \mathrm{C}\right)$ and relative constant humidity $(50 \%-60 \%)$. Animals were treated according to protocols established by the ethics committee of Zhengzhou University, and the in vivo experiments were carried out in accordance with the approved guidelines and approved by the ethics committee of Zhengzhou University. 


\section{Cellular Physiology Cell Physiol Biochem 2018;47:1977-1988 \\ \begin{tabular}{l|l} 
and Biochemistry Published online: July 03, 2018 & $\begin{array}{l}\text { (c) } 2018 \text { The Author(s). Published by S. Karger AG, Basel } \\
\text { www.karger.com/cpb }\end{array}$ \\
\hline
\end{tabular}}

Fan et al.: Bushenshugan Formula Inhibits Lung Cancer

\section{Serum Preparations}

BSF from the First Affiliated Hospital of Zhengzhou University in this study was a Chinese recipe including the following Chinese herbal medicines: Bupleurum 6g, Schisandra 6g, Angelica 6g, Red peony root 6g, Medlar 10g, Dodder 9g, Plantain 15g, Raspberry 9g, Astragalus 18g, Epimedium 9 g, Houttuynia 15g, Trichosanthes 12g, Allium white 9g, Prunella 9g, Ligustrumlucidum 9g, Houttuyniacordata 15 g, Polyporus 9 $\mathrm{g}$, Gentian $2 \mathrm{~g}, 1$ centipede. The concentration of the BSF solutions were $1.25 \mathrm{~g} / \mathrm{mL}$ (low dose) and $3.75 \mathrm{~g} / \mathrm{mL}$ (high dose), and the solutions were stored at $4^{\circ} \mathrm{C}$ for use. DDP was from Shandong Qilu Pharmaceutical Co., Ltd. The 60 male rats were divided into 6 groups using a random digits table method ( $n=10$ per group); the groups included the BSF Low-dose group, BSF High-dose group, cisplatin(DDP) group, Low-dose combined with DDP group, high-dose combined with DDP group and saline group. According to a human and murine body surface area conversion method, the BSF low-dose group and high-dose group were respectively given $15 \mathrm{~g} / \mathrm{kg}$ and $30 \mathrm{~g} / \mathrm{kg}$ BSF by gavage once during 7 days. The DDP group was given BSF solution intraperitoneally at a dose of $8 \mathrm{~g} / \mathrm{kg}$ on the first, third and fifth day. The low-dose combined with DDP group and high-dose combined with DDP group were respectively given $15 \mathrm{~g} / \mathrm{kg}$ and $30 \mathrm{~g} / \mathrm{kg}$ BSF by gavage once a day and $8 \mathrm{~g} / \mathrm{kg}$ DDP on the first, third and fifth day. The saline group received an equal volume of $0.9 \%$ sodium chloride solution orally once during 7days. Chloral hydrate anaesthesia was performed 2 hours after the last gavage, and then, we collected the blood from the abdominal aorta under sterile conditions and separated the serum. The sera were inactivated at $56^{\circ} \mathrm{C}$ for 30 minutes, then filtered by a filter $(0.22 \mu \mathrm{M})$ to remove bacteria, and finally stored at $-20^{\circ} \mathrm{C}$. Drug-containing medium contained $10 \%$ serum from the BSF Low-dose, BSF High-dose, DDP, low-dose combined with DDP, high-dose combined with DDP and saline groups.

\section{Cell lines and cell culture}

The human lung cancer A549 cells were provided by the Cancer Experimental Center of Zhengzhou University. All cells were cultured in RPMI-1640 medium (HyClone, USA)supplemented with 10\% foetal bovine serum(FBS) (HyClone, USA), $100 \mathrm{U} / \mathrm{mL}$ penicillin, and $100 \mu \mathrm{g} / \mathrm{mL}$ streptomycin at $37^{\circ} \mathrm{C}$ in a humidified $5 \% \mathrm{CO}_{2}$ incubator (Thermo, USA).

\section{Cell Proliferation Assay}

A549 cells were seeded in a 96-well culture plate at a density of approximately $4 \times 10^{4}$ cells/well, 6 wells per group. Then, $100 \mu \mathrm{L}$ suspension per well was cultured in 1640 medium containing10\% FBS, and $12 \mathrm{~h}$ later, the medium was discarded, and the cells were treated with $100 \mu \mathrm{L}$ media containing serial concentrations of BSF and cultured for another 72 hours. Cell Counting Kit-8 (CCK-8) solution at 10 $\mu \mathrm{L}$ was added to each well and incubated for another $1 \mathrm{~h}$ before it was discarded. The absorbance(A)was measured by a microplate reader at a wavelength of $450 \mathrm{~nm}$. The inhibitory ratio was calculated by the following formula: Inhibition rate $(\%)=(1$-experimental group $\mathrm{A} /$ normal group $\mathrm{A}) \times 100 \%$. The experiment was repeated three times under the same conditions.

\section{Colony formation assay}

A549 cells were trypsinised and seeded at 1000 cells per well in 6-well dishes. Cells were allowed to attach overnight and then exposed to different concentrations of BSF for 14 days. After they were fixed with $4 \%$ paraformaldehyde, the cells were stained with $0.1 \%$ crystal violet. The colony formation rate was calculated and analysed as the following equation: colony formation rate $=$ (number of colonies $/$ number of seeded cells) $\times 100 \%$.

\section{Migration assay}

To determine cell motility, A549 cells $\left(1 \times 10^{5}\right.$ cells/well $)$ were seeded in 24 -well plates and incubated at $37^{\circ} \mathrm{C} / 5 \% \mathrm{CO}_{2}$ overnight. Wounds were made using $200 \mu \mathrm{L}$ pipette tips. Cells were then washed twice with Hank's balanced salt solution to remove floating cells, and fresh medium was added. Cells migrating from the edge of the wound were photographed from five random fields of view during different time periods.

Transwell Invasion Assay

The invasion assays with A549 cells were performed as described previously. The invasion of the cells was measured with Matrigel-coated transwell inserts. Matrigel matrix (25 $\mu \mathrm{L} /$ well) was placed in 
the upper chamber of the transwell filter (pore size of $8 \mu \mathrm{m}$ ) and was incubated for 30 minutes at $37^{\circ} \mathrm{C}$ for gelling. A549 cells were trypsinised and seeded at $2 \times 10^{4}$ cells $(300 \mu \mathrm{L})$ per well in basic 1640 medium with Matrigel matrix $(25 \mu \mathrm{L} /$ well) in the upper chambers. Drug-containing medium was placed in the lower well. After $24 \mathrm{~h}$ incubation at $37^{\circ} \mathrm{C}$, cells in the upper side were scraped with a cotton swab, and invaded cells were fixed with $4 \%$ paraformaldehyde and stained with Giemsa. The cells were rinsed with PBS, air-dried and observed under a microscope equipped with a camera.

\section{Spheroid body formation}

A549 cells (1000 cells/mL) were seeded on ultra-low attachment plates (Corning, Lowell, MA, USA) and incubated at $37^{\circ} \mathrm{C} / 5 \% \mathrm{CO}_{2}$ to form spheroid bodies. Cells were cultured in DMEM/F12 (Gibco, Carlsbad, CA, USA)with B-27 supplements (Gibco), 10\% FBS, basic fibroblast growth factor (Sigma-Aldrich, Poole, UK) and epidermal growth factor (Calbiochem, San Diego, CA, USA) for 7 days at $37^{\circ} \mathrm{C} / 5 \% \mathrm{CO}_{2}$.

\section{Cell Cycle Assay}

Cells were seeded in 24-well plates at a density of approximately $1 \times 10^{5}$ cells/well and incubated for $24 \mathrm{~h}$, and then, the medium was discarded, and cells were cultured with the drug-containing medium for $72 \mathrm{~h}$. A549 cells were digested with trypsin without EDTA and collected in a $1.5 \mathrm{~mL}$ centrifuge tube by centrifugation at $1000 \mathrm{rpm} / \mathrm{min}$. After washing twice with cold saline and discarding the supernatant, $1 \mathrm{~mL}$ precooled $70 \%$ ethanol was added at $4^{\circ} \mathrm{C}$ overnight. The next day, the cells were resuspended in $500 \mu \mathrm{L}$ precooled phosphate buffered saline (PBS) containing 25g/L RNase and 2.5 $\mathrm{L}$ PI (10 g/L) for staining at $37^{\circ} \mathrm{C}$ for $30 \mathrm{~min}$. Cell cycle analysis was performed by flow cytometry, and the cells in the G0/G1, Sand G2/M phases were measured using FlowJo software.

\section{Cell Apoptosis Assay}

The cells were digested with trypsin without EDTA. The density of cells was adjusted to $1.0 \times 10^{5} / \mathrm{ml}$, and $1 \mathrm{~mL}$ of cells was seeded in each well of 24-well plates. BSF-induced apoptosis of A549 cells was quantified by an Annexin V-FITC/PI dual staining assay [14]. Briefly, cells were treated with different concentrations of BSF for $72 \mathrm{~h}$. Then, the cells were collected, centrifuged and resuspended with PBS. After centrifugation, the cells were resuspended with $500 \mu \mathrm{L}$ binding buffer and incubated with $5 \mu \mathrm{LAnnexin} \mathrm{V-FITC}$ and $4 \mu \mathrm{LPI}$ at $4^{\circ} \mathrm{C}$ for $0.5 \mathrm{~h}$ in the dark. Then, the cells were analysed immediately by flow cytometry.

\section{RNA extraction, cDNA synthesis, and quantitative real-time PCR}

After collecting A549 cells treated for $72 \mathrm{~h}$, total RNA was extracted using TRIzol (Invitrogen, USA). The first-strand cDNA was synthesised from $1 \mu \mathrm{g}$ of total RNA using a Super Quick RT cDNA Kit (CWBIO, China) according to the manufacturer's instructions. Then, quantitative real-time polymerase chain reaction(qRTPCR) analysis was performed using SYBR Green PCR Master Mix (Qiagen, Germany) and assessed by Agilent Mx3005P. GAPDH was used as an internal control. Primer sequences for qRT-PCR are shown in Table 1.

\section{Westernblot}

First, $1 \times 10^{6}$ cells were lysed with mixed RIPA buffer containing $50 \mathrm{mM}$ Tris- $\mathrm{HCl}(\mathrm{pH} 7.5), 150 \mathrm{mM} \mathrm{NaCl}$, $1 \%$ NP-40, 0.5\% sodium deoxycholate, $0.1 \%$ SDS and 1\%PMSF(100×)(Cwbio, Beijing, China) on ice for approximately $30 \mathrm{mins}$ and then centrifuged at $1500 \mathrm{~g}$ for five min at $4^{\circ} \mathrm{C}$. Next,30 $\mu \mathrm{g}$ proteins were loaded onto a $12 \%$ SDS-PAGE gel and transferred to a PVDF (Millipore, Bedford, MA) membrane. The membrane was blocked in TBST buffer containing $5 \%$ non-fat milk at room temperature $\left(22^{\circ} \mathrm{C}\right)$ for $1 \mathrm{~h}$ and then probed with $\operatorname{Bcl} 2$ (1:1000, Genetex, GTX34425), P53(1:500, Abcam, ab131442), Bax2(1:1000, Genetex, GTX109683), CyclinD1(1:3000, Proteintech, 60186-1-lg), E-cadherin(1:2000, Abcam, ab40772), N-cadherin(1:800, Abcam, ab18203), Vimentin(1:1000, Abcam, ab92547), $\alpha$-SMA(1:1000, Genetex, GTX100034), snail(1:1000, Abcam, ab53519), P-PI3K(1:1000, CST,13857), PI3K(1:1000, CST,4249), P-AKT(1:1000, CST, 4691),

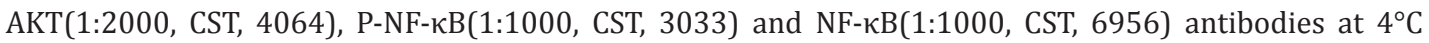


overnight, followed by incubation with the appropriate horseradish peroxidase-conjugated secondary antibodies for $1 \mathrm{~h}$ at room temperature. Then, the membrane was exposed to chemiluminescent reagent (ECL) for approximately 5-10 min. The membranes were exposed to X-ray photographic films in a darkroom, and the band densities were later quantified with Image J software (NIH, USA).

\section{Statistical Analysis}

All data are expressed as the mean \pm standard deviation(SD) of three independent biological experiments. Statistical significance was assessed using the two tailed Student's t-test(for comparisons of two treatment groups) and one-way ANOVA(for comparisons of three or more groups). ${ }^{*} \mathrm{P}<0.05$ was considered statistically significant.

\section{Results}

\section{Effects of the BSF serum on proliferation and tumourigenicity of A549 cells}

All groups showed significantly inhibited proliferation of A549 lung cancer cells compared with the saline group (Fig. 1A).The inhibition rate of the BSF High-dose group was second only after the DDP group. The inhibition rate of the high-dose combined with DDP group was the strongest, and after treatment for $24 \mathrm{~h}, 48 \mathrm{~h}$, and $72 \mathrm{~h}$, the inhibitory rates were $45.39 \%, 54.76 \%$ and $59.94 \%$, respectively.

Abnormal proliferation of cancer cells results in malignant tumours. Colony formation assays directly assess the proliferative ability of a single cancer cell to form a cell colony in vitro. The tumourigenicity of A549 cells and the inhibitory effect of BSF on colony formation of A549 cells were measured by colony formation assays. BSF effectively inhibited the colony-forming ability of DDP-treated A549 cells compared with those treated with cisplatin alone. The colony formation ability decreased drastically in the high-dose BSF combined with DDP group. Representative images and quantitative analysis of the colony formation rate are presented in Fig. 1B and C.

\section{Effects of the BSF serum on apoptosis and the cell cycle of A549 cells}

To investigate the cytotoxic mechanism of BSF, we determined the effects on A549 cell apoptosis by using flow cytometry. The results of apoptosis assays are shown in Fig. 2A and B. BSF was found to be a potent inducer of apoptosis in a dose-dependent manner. The apoptosis rate of the high-dose combined with DDP group was the highest among all the groups.

The cell cycle involves a series of events that lead to cell division and replication. The cell cycle consists of four distinct phases: G1 phase, S phase(synthesis), G2 phase (collectively known as interphase) and $M$ phase(mitosis).As a hallmark of A549 cells, cell cycle dysregulation contributes to the aberrant cell proliferation and development of cancer. Therefore, targeting cell cycle progression is an effective method for novel anti-tumour drug discovery. To determine whether the

Fig. 1. Effects of the BSF serum on the proliferation and tumourigenicity of A549 cells.(A)Cell viability was evaluated at the indicated time points using CCK8 assays.(B) The colonies of A549 cells with $>100$ cells were quantified. Original magnification:200×. (C)The bar graphs represent the average number of cloned cells under each group of processing conditions: group a. Saline group, b. BSF Low-dose group, c. BSF High-dose group, d. Low-dose combined with DDP, e. High-dose combined with DDP, f. DDP. The data are expressed as the mean value \pm SD. ${ }^{*} \mathrm{P}<0.05$ compared with group a.

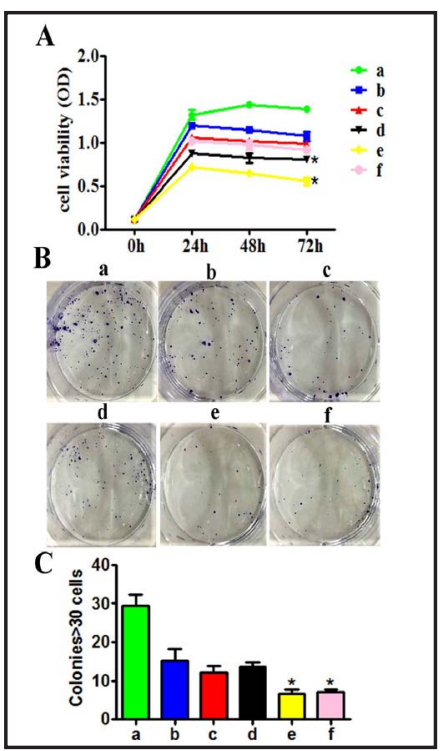


Fig. 2. Effects of the BSF serum on the apoptosis and cell cycle of A549 cells. (A)Cell apoptosis was measured by flow cytometric analysis. (B)The bar graphs represent the percentage of apoptotic cells. (C) The cell cycle was measured by flow cytometric analysis. (D) The bar graphs represent the percentage of G2/M, S and G0/G1 phase A549 cells in each group.(E)The expression levels of the cell apoptosis and cell cycle-related proteins Bcl-2, P53, Bax and CyclinD1 were detected by western blot.(F) The bar graphs represent the average quantitative value of normalized expression to $\beta$-Actin in each group: group a. Saline group, b. BSF Low-dose group, c. BSF High-dose group, d. Low-dose combined with DDP, e. High-dose combined with DDP, f. DDP. The data are expressed as the mean value $\pm \mathrm{SD}$. ${ }^{*} \mathrm{P}<0.05$ compared with group a.

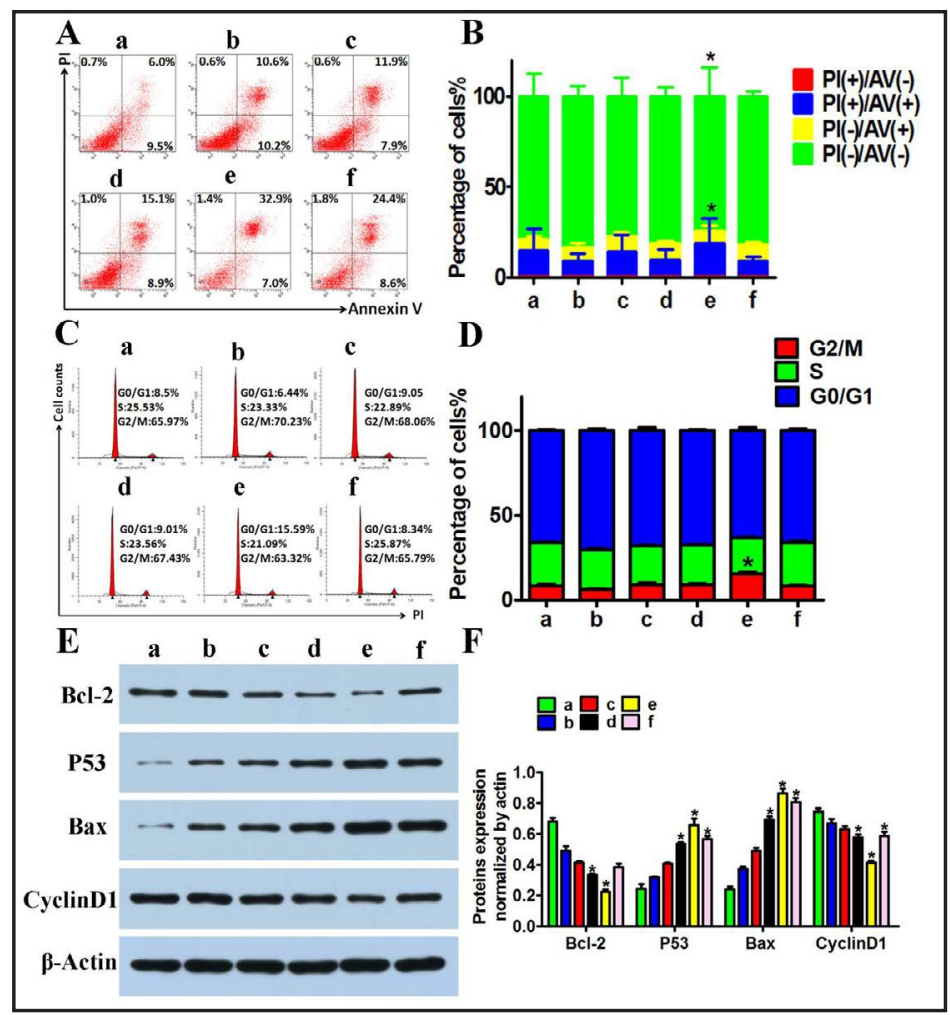

inhibitory effects of BSF on A549 cell growth were correlated with cell cycle arrest, we further investigated the effects of BSF on cell cycle progression. As shown in Fig. 2C, highdose BSF combined with DDP could induce cell cycle arrest in the G2/M phase in A549 cells.

Some apoptotic and cell cycle regulatory proteins were also detected by western blot analyses (Fig. 2E and 2F). The expression levels of Bcl-2 and CyclinD1 were inhibited by BSF, and the high-dose BSF combined with DDP treatment was the most effective. However, BSF could increase the expression ofBax and P53, and DDP had a synergistic effect.

\section{Effects of the BSF serum on the cancer stem cells (CSCS) of A549 cells}

Cancer stem cells(CSCs) are a specific subpopulation of tumour cells that selectively possess tumour initiation and self-renewal capacities, as well as the ability to give rise to bulk populations of non-tumourigenic cancer cell progeny through differentiation [15]. We examined the CSC properties of A549 cells by BSF. We generated floating spheroid bodies to assess the self-renewal and differentiation capacities of these cells. As shown in Fig. 3A and $\mathrm{B}$, BSF altered the morphology of spheroid cells, and the sizes of the spheroid bodies were reduced. These results suggest that BSF strongly inhibited CSC properties of A549 cells.

\section{Effects of the BSF serum on the migration and invasion of A549 cells}

The migratory effects of BSF against A549 cells were analysed by wound-healing assays according to the previous method. Wounds were made using pipette tips, and cell motility was observed after $24 \mathrm{~h}$, as shown in Fig. $4 \mathrm{~A}$ and B. Based on the results, high-dose BSF combined with DDP significantly reduced cell motility of A549 cells. For wound-healing assays, phase contrast microscopy images of control A549 cells treated with BSF cultures were acquired at 0 and $24 \mathrm{~h}$ after wounding. The high-dose BSF combined with DDP group showed significantly reduced cell motility of A549 cells.

BSF inhibited the invasion of A549 cells. After cells were cultured with drug-containing medium for $24 \mathrm{~h}$, the invasion of A549 cells showed different degrees of decline in Fig. 5A and B. While the BSF high-dose group showed significantly inhibited invasion of A549 cells, 
Fig. 3. Effects of the BSF serum on the cancer stem cells of A549 cells. (A)Bright-field microscopy images of spheres generated from A549 cells after the sphere-formation assay. Original magnification: $200 \times$. (B)The bar graphs represent the number of spheres in each group: group a. Saline group, b. BSF Low-dose group, c. BSF High-dose group, d. Low-dose combined with DDP, e. High-dose combined with DDP, f. DDP. The data are expressed as the mean value $\pm \mathrm{SD} .{ }^{*} \mathrm{P}<0.05$ compared with group a.

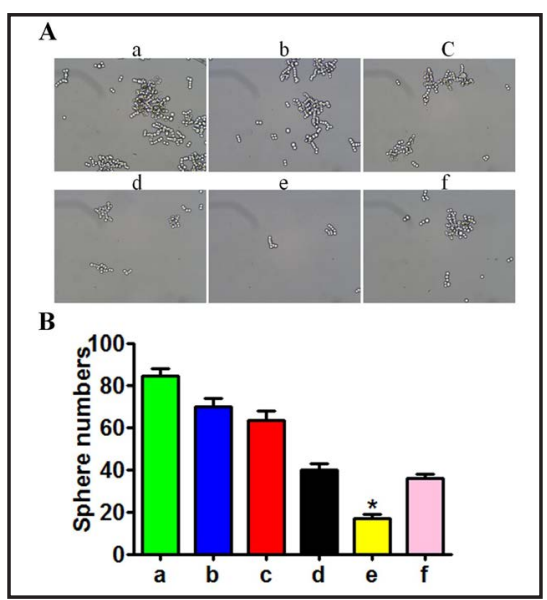

Fig. 4. Effects of the BSF serum on the migration of A549 cells. The migratory capabilities of A549 cells were evaluated by scratch assays. (A)Representative microscopic images of the bottom chamber are shown. (B) The bar graphs represent the fold change of cell migration distance in each group: group a. Saline group, b. BSF Low-dose group, c. BSF High-dose group, d. Lowdose combined with DDP, e. High-dose combined with DDP, f. DDP. The data are expressed as the mean value \pm SD. ${ }^{*} \mathrm{P}<0.05$ compared with group a.

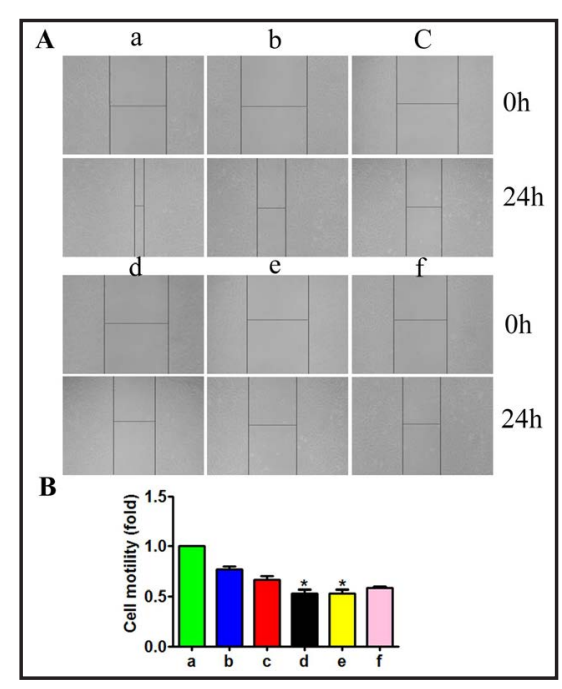

Fig. 5. Effects of the BSF serum on the invasion of A549 cells. The invasive capabilities of A549 cells were evaluated by using transwell invasion assays. (A)Representative microscopic images of the bottom chamber are shown. (B)The bar graphs represent the average number of invaded cells on the underside of the membrane in each group: group a. Saline group, b. BSF Low-dose group, c. BSF High-dose group, d. Low-dose combined with DDP, e. High-dose combined with DDP, f. DDP. The data are expressed as the mean value $\pm \mathrm{SD}$. ${ }^{*} \mathrm{P}<0.05$ compared with group a.

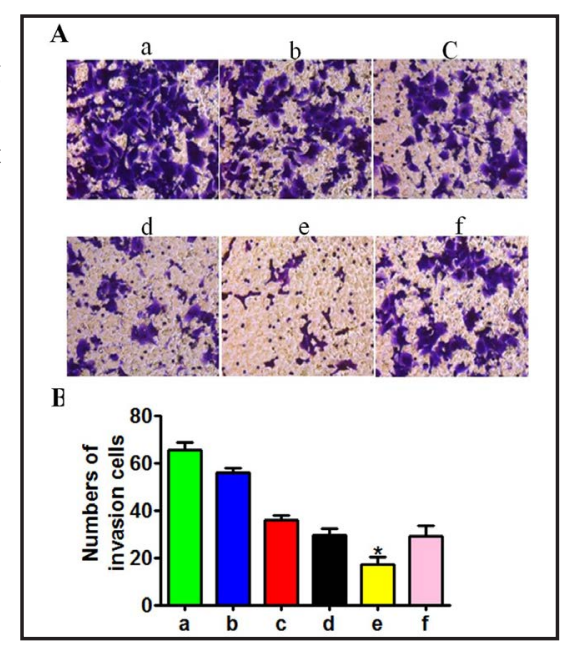

this treatment showed synergy combined with DDP. These results also suggested that DDP might have synergistic effects on the inhibition of invasion. The differences between the experimental groups and control group were significant $(\mathrm{P}<0.05)$.

\section{Effects of the BSF serum on EMT of A549 cells and related molecular mechanisms}

As shown in Fig. 6A, the DDP group showed no significant inhibitory effects on the EMT-related genes, but BSF in this assay decreased the expression levels of N-cadherin and KARGER 
Fig. 6. (A)Effects of the BSF serum on genes related to EMT. Ecadherin, N-cadherin, Vimentin, $\alpha$-SMA and Snail were decreased at the mRNA level. Relative gene expression was determined by normalising the expression of each target gene to GAPDH. (B)The expression levels of the EMT-related proteins E-cadherin, $\mathrm{N}$-cadherin, Vimentin, $\alpha$-SMA and Snail were examined through western blot. (C)The bar graphs represent the average quantitative value of normalized expression to $\beta$-Actin in each group. (D)The expression levels of PI3K-AKT-NF/КB-related proteins.PI3K, P-PI3K, AKT, P-AKT, $\mathrm{NF} / \kappa \mathrm{B}$ and $\mathrm{P}-\mathrm{NF} / \kappa \mathrm{B}$ were examined through western blot. (E) The bar graphs represent the average quantitative value of normalized expression to $\beta$-Actin in each group: group a. Saline group, b. BSF Low-dose group, c. BSF High-dose

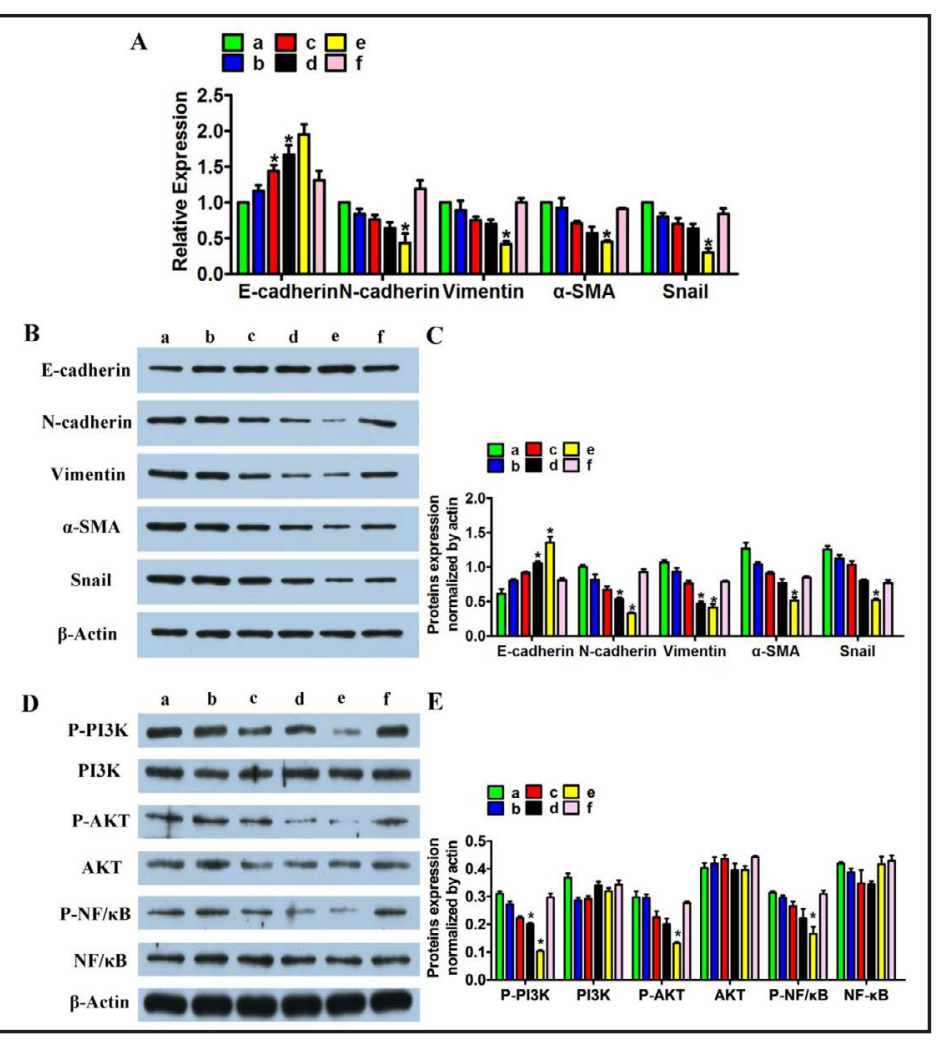
group, d. Low-dose combined with DDP, e. High-dose combined with DDP, f. DDP.The data are expressed as the meanvalue \pm SD. ${ }^{*} \mathrm{P}<0.05$ compared with group a.

Vimentin and increased the expression of E-cadherin in a concentration-dependent manner. Importantly, BSF combined with DDP exhibited the most potent regulatory effect on target genes. The protein expression levels are shown in Fig. 6B and Fig. 6C. BSF could increase the expression of E-cadherin and decrease the expression levels of $\mathrm{N}$-cadherin, Vimentin, $\alpha$-SMA and Snail. All these results demonstrated that DDP displayed synergy with BSF in the EMT progression. To explore the molecular mechanisms related to EMT of A549 cells, we examined the expression levels of proteins related to the PI3K-AKT signalling pathway. As shown in Fig. 6D and Fig. 6E, BSF combined with DDP could decrease the expression levels of P-PI3K, P-AKT and P-NF- $\mathrm{BB}$.

\section{Discussion}

NSCLC is one of the most common malignant tumours and a leading cause of cancerrelated death worldwide [16]. Although the National Comprehensive Cancer Network(NCCN) panel recommended platinum-based chemotherapy regimens as a preferred therapy for patients with NSCLCs, these treatments have many side effects, including fever, anaemia, and alopecia, which have significantly limited their use and decreased patient quality of life [17]. Since metastasis and recurrence are considered to be responsible for the failure of treatments, the mechanism of lung cancer metastasis has been a hot spot in cancer research $[18,19]$. Therefore, there is an urgent and increasing need to develop effective novel methods or pharmacotherapies to solve this problem.

EMT is an evolutionarily conserved process that plays an important role in the embryonic development and the progression of tumours. Meanwhile, EMT is believed to be involved in metastasis and drug resistance [3]. A recent study also suggested that EMT could increase 
the motility and invasiveness of cancer cells [20-22]. E-cadherin and N-cadherin are major biological markers in EMT progression, and they could be regulated during the therapeutic process $[20,22,23]$. In addition, Vimentin is widely used as a marker of the epithelial to mesenchymal transitions (EMTs) that occur during embryogenesis and metastasis [24, 25]. As a member of the zinc finger inhibitory transcription factor family, Snail could bind directly to the promoter region of E-cadherin, thus inhibiting the expression levels of E-cadherin and then promoting EMT $[26,27]$. The PI3K/AKT signalling pathway mediates the process of EMT and has attracted widespread attention as a potential target for the prevention and treatment of metastatic tumours $[28,29]$. NF- $\kappa \mathrm{B}$ is a ubiquitously expressed transcription factor that has been reported to act downstream of the PI3K/Akt pathway. This protein is a major target of activated AKT that regulates genes involved in cancer cell migration and invasion [30].

Cell apoptosis and the cell cycle play an important role in the pathogenesis of cancers. The development of cancer mainly depends on the balance of pro-apoptotic proteins, such as Bax, and anti-apoptotic proteins, such as Bcl-2 [31].The p53 protein is a regulatory factor of many processes necessary for the proper functioning of cells. It plays a critical role as a tumour suppressor, inducing cell cycle arrest and apoptosis [32].CyclinD1,as a major member of the cyclins, plays an important role in cell cycle progression [33].

Lung cancer according to TCM is a disease that is involves excess in the body but deficiency in the holistic concept of TCM, and the deficiency is the root cause, while the excess is the manifestation. Lung cancer lesions are located in the lungs but are often related to the liver, spleen, stomach and kidney. Liver governs conveyance and dispersion and affects emotions. Lung governs the qi, whose diffusion and decrease depend on the conveyance and dispersion functions of the liver. Lung is the mother of the kidney and governs regulation of water passages, and kidney governs water metabolism. In early research, we found that the kidney regulates the lung via androgens and their receptor, which may be the material basis of the mutual relationship of the lung and kidney [34]. In the late stage of lung cancer, symptoms include kidney essence deficiency and emotional depression. Studies demonstrated that Bushen prescription can improve the patient's immune system $[35,36]$ and inhibit lung cancer growth and metastasis through a variety of mechanisms $[9,37]$. Dispersing stagnated liver qi to regulate qi for the treatment of lung cancer is still rare, but the incidence of depression in the patients who were diagnosed with lung cancer is approximately 15\%-44\%according to statistics [38].

With broad applications in the treatment of lung cancer [39, 40], breast cancer [41], colorectal cancer [42] and other tumours, traditional Chinese medicines have been explored and have provided new therapeutic targets. BSF had beneficial effects on the kidney and the liver in clinical practice. BSF was also shown to benefit the kidney and nourish semen, dispersing stagnated liver qi to regulate qi, dissipating phlegm and resolving masses. In our earlier research, BSF contributed to the improvement of human immunity and inhibited proliferation of tumour cells by apoptosis. However, the effect of BSF on EMT remains unknown.

In this work, BSF could effectively inhibit the proliferation of A549 cells in time- and dose-dependent manners and had synergistic effects combined with DDP. The inhibition rates at $24 \mathrm{~h}, 48 \mathrm{~h}$ and $72 \mathrm{~h}$ were $45.39 \%, 54.76 \%$ and $59.94 \%$, respectively. The colony formation ability decreased drastically with high-dose BSF combined with DDP. Annexin V/ PI staining with flow cytometry suggested that the herb serum could induce cell apoptosis in the concentration-dependent manner and arrest the cell cycle at G2/M phase. Western blot analysis showed that BSF combined with DDP could decrease the expression levels of cyclinD1 and Bcl2 but increased the expression levels of P53 and Bax. Floating spheroid bodies demonstrated that BSF strongly inhibited the CSC properties ofA549 cells. In further research, BSF decreased the gene and protein expression levels of N-cadherin, Vimentin, Snail and $\alpha$-SMA, while it increased the expression levels of E-cadherin. Further, BSF combined with DDP could decrease the protein expression levels of P-PI3K, P-AKT and NF-кB. 
In summary, BSF could inhibit the proliferation, decrease the colony formation, induce cell apoptosis, inhibit the invasion and block the CSC properties of A549 cells. Cellular mechanisms suggested that BSF inhibited the process of EMT and affected the expression of N-cadherin, Vimentin, Snail, $\alpha$-SMA and E-cadherin through PI3K/AKT/NF- $\kappa B$ pathway. Thus, BSF as a traditional Chinese medicine might provide new insight into the treatment of NSCLC. In addition, elucidation of the pharmacological mechanisms and the specific therapeutic targets of BSF is underway.

\section{Acknowledgements}

This study was supported by grants from the National Natural Science Foundation of China (Grant No.71673254, 81473497), The National Key Research and Development Program of China-The construction and promotion of the demonstration system of based on telemedicine/Mhealth network (Grant No.2017YFC0909900), Program of Science \& Technology of Henan Province (Grant No.201602037), the Innovation Team of the First Affiliated Hospital of Zhengzhou University.

Jie Zhao and Shuling Wang conceptualised and designed this research. Zhirui Fan, Mengmeng Dou, Wenhua Xue, Jingli Lu and Lifeng Li performed the experiments and wrote the paper. Mingzhi Zhang, Xiaoming Deng and Yunkai Zhai reviewed and edited the manuscript. All authors read and approved the manuscript. The authors declare that they have no conflicts of interest.

\section{Disclosure Statement}

The authors declare that they have no competing interests.

\section{References}

1 Sangodkar J, Katz S, Melville H, Narla G: Lung adenocarcinoma: lessons in translation from bench to bedside. Mt Sinai J Med 2010;77:597-605.

-2 Ettinger DS, Wood DE, Akerley W, Bazhenova LA, Borghaei H, Camidge DR, Cheney RT, Chirieac LR, D’Amico TA, Dilling TJ, Dobelbower MC, Govindan R, Hennon M, Horn L, Jahan TM, Komaki R, Lackner RP, Lanuti M, Lilenbaum R, Lin J, Loo BW, Jr., Martins R, Otterson GA, Patel JD, Pisters KM, Reckamp K, Riely GJ, Schild SE, Shapiro TA, Sharma N, Stevenson J, Swanson SJ, Tauer K, Yang SC, Gregory K, Hughes M: NCCN Guidelines Insights: Non-Small Cell Lung Cancer, Version 4.2016 J Natl Compr Canc Netw 2016;14:255-264.

3 Xiao D, He J: Epithelial mesenchymal transition and lung cancer. J Thorac Dis 2010;2:154-159.

-4 Ren J, Chen Y, Song H, Chen L, Wang R: Inhibition of ZEB1 reverses EMT and chemoresistance in docetaxelresistant human lung adenocarcinoma cell line. J Cell Biochem 2013;114:1395-1403.

5 Wang T, Guo R, Zhou G, Zhou X, Kou Z, Sui F, Li C, Tang L, Wang Z: Traditional uses, botany, phytochemistry, pharmacology and toxicology of Panax notoginseng (Burk.) F.H. Chen: A review. J Ethnopharmacol 2016;188:234-258.

6 Chen XZ, Cao ZY, Liao LM, Liu ZZ, Du J: Application of serum pharmacology in evaluating the antitumor effect of Fuzheng Yiliu Decoction from Chinese medicine. Chin J Integr Med 2014;20:450-455.

7 Liao YH, Li CI, Lin CC, Lin JG, Chiang JH, Li TC: Traditional Chinese medicine as adjunctive therapy improves the long-term survival of lung cancer patients. J Cancer Res Clin Oncol 2017;10.1007/s00432-017-2491-6

8 Chuang MH, Jan MS, Chang JT, Lu FJ: The Chinese medicine JC-001 enhances the chemosensitivity of Lewis lung tumors to cisplatin by modulating the immune response. BMC Complement Altern Med 2017;17:210.

-9 Zhu X, Zhou Y, Xu Q Wu J: Traditional Chinese medicine Jianpi Bushen therapy suppresses the onset of premetastatic niche in a murine model of spontaneous lung metastasis. Biomed Pharmacother 2017;86:434440. 
10 Liu Y, Wang S: Clinical effect observation of Bushen Sanjie Decoction combined with chemotherapy of GP regimen in treating patients with advanced non-samll cell lung cancer. China Journal of Traditional Chinese Medicine and Pharmacy 2011;26:864-867.

11 Deng X, Fan Z, Miao X, Li L, Wang S, Zhan X: Effects of Bushen Shugan Formula on Human Lung Cancer A549 Cells and Related Stem Genes. Chinese Journal of Experimental Traditional Medical Formulae 2015;21:136-140.

12 Shang Y, Wang S: Inhibiting Effect and Mechanism of Drug-contained Serum of Bushen Shugan Formula on A549 Cells Proliferation and Metastasis by PTEN-PI3K-AKT Pathway. Chinese Journal of Experimental Traditional Medical Formulae 2015;21:153-157.

13 Miao X, Wang S, Gu Z, Du J: Research about inhibition effects of Bushen Shugan Formula on human lung adenocarcinoma A549 cells and apoptosis related genes. China Journal of Traditional Chinese Medicine and Pharmacy 2014;29:2661-2664.

14 Gao W, Liu Y, Qin R, Liu D, Feng Q: Silence of fibronectin 1 increases cisplatin sensitivity of non-small cell lung cancer cell line. Biochem Biophys Res Commun 2016;476:35-41.

15 Abdel-Aziz HA, Ghabbour HA, Eldehna WM, Al-Rashood ST, Al-Rashood KA, Fun HK, Al-Tahhan M, AlDhfyan A: 2-((Benzimidazol-2-yl)thio)-1-arylethan-1-ones: Synthesis, crystal study and cancer stem cells CD133 targeting potential. Eur J Med Chem 2015;104:1-10.

16 Miller KD, Siegel RL, Lin CC, Mariotto AB, Kramer JL, Rowland JH, Stein KD, Alteri R, Jemal A: Cancer treatment and survivorship statistics, 2016 CA Cancer J Clin 2016;66:271-289.

17 Wood DE, Kazerooni E, Baum SL, Dransfield MT, Eapen GA, Ettinger DS, Hou L, Jackman DM, Klippenstein D, Kumar R, Lackner RP, Leard LE, Leung AN, Makani SS, Massion PP, Meyers BF, Otterson GA, Peairs K, Pipavath S, Pratt-Pozo C, Reddy C, Reid ME, Rotter AJ, Sachs PB, Schabath MB, Sequist LV, Tong BC, Travis WD, Yang SC, Gregory KM, Hughes M, National comprehension cancer n: Lung cancer screening, version 1.2015: featured updates to the NCCN guidelines. J Natl Compr Canc Netw 2015;13:23-34; quiz 34.

18 Cheng ZH, Shi YX, Yuan M, Xiong D, Zheng JH, Zhang ZY: Chemokines and their receptors in lung cancer progression and metastasis. J Zhejiang Univ Sci B 2016;17:342-351.

19 Su JM, Hsu YY, Lin P, Chang H: Nuclear Accumulation of Heat-shock Protein 90 Is Associated with Poor Survival and Metastasis in Patients with Non-small Cell Lung Cancer. Anticancer Res 2016;36:2197-2203.

20 Shi H, Ji Y, Zhang D, Liu Y, Fang P: MiR-135a inhibits migration and invasion and regulates EMT-related marker genes by targeting KLF8 in lung cancer cells. Biochem Biophys Res Commun 2015;465:125-130.

21 Yang H, Zhan L, Yang T, Wang L, Li C, Zhao J, Lei Z, Li X, Zhang HT: Ski prevents TGF-beta-induced EMT and cell invasion by repressing SMAD-dependent signaling in non-small cell lung cancer. Oncol Rep 2015;34:87-94.

22 You J, Li Y, Fang N, Liu B, Zu L, Chang R, Li X, Zhou Q: MiR-132 suppresses the migration and invasion of lung cancer cells via targeting the EMT regulator ZEB2 PLoS One 2014;9:e91827.

23 Zhang X, Liu G, Kang Y, Dong Z, Qian Q, Ma X: N-cadherin expression is associated with acquisition of EMT phenotype and with enhanced invasion in erlotinib-resistant lung cancer cell lines. PLoS One 2013;8:e57692.

24 Kidd ME, Shumaker DK, Ridge KM: The role of vimentin intermediate filaments in the progression of lung cancer. Am J Respir Cell Mol Biol 2014;50:1-6.

-25 Mendez MG, Kojima S, Goldman RD: Vimentin induces changes in cell shape, motility, and adhesion during the epithelial to mesenchymal transition. FASEB J 2010;24:1838-1851.

-26 Abd El-Rehim DM, Abd-Elghany MI, Nazmy MH: Integrin-Linked Kinase, Snail and Multidrug Resistance Protein 1: Three concordant players in the progression of non-small cell lung cancer. J Egypt Natl Canc Inst 2015;27:129-137.

-27 Wu Y, Zhou BP: TNF-alpha/NF-kappaB/Snail pathway in cancer cell migration and invasion. Br J Cancer 2010;102:639-644.

28 Bakin AV, Tomlinson AK, Bhowmick NA, Moses HL, Arteaga CL: Phosphatidylinositol 3-kinase function is required for transforming growth factor beta-mediated epithelial to mesenchymal transition and cell migration. J Biol Chem 2000;275:36803-36810.

-29 Xu Q, Ma J, Lei J, Duan W, Sheng L, Chen X, Hu A, Wang Z, Wu Z, Wu E, Ma Q Li X: alpha-Mangostin suppresses the viability and epithelial-mesenchymal transition of pancreatic cancer cells by downregulating the PI3K/Akt pathway. Biomed Res Int 2014;2014:546353. 


\section{Cellular Physiology Cell Physiol Biochem 2018;47:1977-1988 \begin{tabular}{l|l} 
DOI: 10.1159/000491466 & O 2018 The Author(s). Published by S. Karger AG, Basel \\
www.karger.com/cpb
\end{tabular} \\ Fan et al.: Bushenshugan Formula Inhibits Lung Cancer}

30 Ko BS, Chang TC, Chen CH, Liu CC, Kuo CC, Hsu C, Shen YC, Shen TL, Golubovskaya VM, Chang CC, Shyue SK, Liou JY: Bortezomib suppresses focal adhesion kinase expression via interrupting nuclear factor-kappa B. Life Sci 2010;86:199-206.

31 Adams JM, Cory S: The Bcl-2 apoptotic switch in cancer development and therapy. Oncogene 2007;26:1324-1337.

-32 Guadagno J, Swan P, Shaikh R, Cregan SP: Microglia-derived IL-1beta triggers p53-mediated cell cycle arrest and apoptosis in neural precursor cells. Cell Death Dis 2015;6:e1779.

-33 Deng M, Zeng C, Lu X, He X, Zhang R, Qiu Q, Zheng G, Jia X, Liu H, He Z: miR-218 suppresses gastric cancer cell cycle progression through the CDK6/Cyclin D1/E2F1 axis in a feedback loop. Cancer Lett 2017;403:175-185.

34 Wang S: Material Basis of the Mutual Relationship of Lung and Kidney. Chinese Journal of Basic Medicine in Traditional Chinese Medicine 2002;8:9.

35 Zhou YB, Yu JE, Wu J, Bai L, Huo LL, Zhang XG, Li LQ: [Effects of Chinese herbal medicine Bushen Gubiao Recipe on toll-like receptor 4 and CD4(+)CD25(+)foxp3(+) regulatory T cells in mice with recurrent respiratory tract infections]. Zhong Xi Yi Jie He Xue Bao 2010;8:1053-1059.

-36 Wang YJ, Shi Q, Jiang JC, Bian Q, Liang QQ Li CG, Zhou Q, Cui XJ, Lu S, Zhou CJ: [Mechanism of Yiqi Huayu Bushen recipe in treating cervical syndrome in rats with qi deficiency, blood stasis and kidney deficiency]. Zhong Xi Yi Jie He Xue Bao 2009; 7:53-58.

-37 Cao Y, Xia QH, Meng H, Zhong AP: Pharmacological effects of serum containing chinese medicine Bushen Huayu Jiedu Compound Recipe in lung cancer drug-resistance cells. Chin J Integr Med 2008;14:46-50.

38 Hopwood P, Stephens RJ: Depression in patients with lung cancer: prevalence and risk factors derived from quality-of-life data. J Clin Oncol 2000;18:893-903.

-39 Chai XS, Zhang XX, Wu WY: Xiaoji Decoction inhibited cell proliferation and induced apoptosis through Akt signaling pathway in human lung cancer A549 cells. Chin J Integr Med 2014;20:701-705.

40 Wang SF, Wang Q, Jiao LJ, Huang YL, Garfield D, Zhang J, Xu L: Astragalus-containing Traditional Chinese Medicine, with and without prescription based on syndrome differentiation, combined with chemotherapy for advanced non-small-cell lung cancer: a systemic review and meta-analysis. Curr Oncol 2016;23:e188195.

41 Su K, Hu P, Wang X, Kuang C, Xiang Q, Yang F, Xiang J, Zhu S, Wei L, Zhang J: Tumor suppressor berberine binds VASP to inhibit cell migration in basal-like breast cancer. Oncotarget 2016;7:45849-45862.

42 Sun G, Wei L, Feng J, Lin J, Peng J: Inhibitory effects of Hedyotis diffusa Willd. on colorectal cancer stem cells. Oncol Lett 2016;11:3875-3881. 